\title{
Child forensic interviewing - An important investigative tool in child sexual abuse cases
}

\author{
Senthil Kumar $\mathbf{R}^{1^{*}}$, Y.S Bansal ${ }^{2}$, N.C Priyadarshini ${ }^{3}$
}

${ }^{\mathbf{1}}$ Assistant Professor, ${ }^{\mathbf{2}}$ Professor, ${ }^{\mathbf{3}}$ Senior Resident, ${ }^{\mathbf{1 , 2}}$ Dept. of Forensic Medicine, ${ }^{\mathbf{3}}$ Dept. of Psychiatry, ${ }^{\mathbf{1 , 2}}$ Postgraduate Institute of Medical Education and Research, Chandigarh, Punjab, ${ }^{3}$ UCMS and GTB Hospital, Delhi, India

\section{*Corresponding Author: Senthil Kumar R}

Email: drsenthilpgimer@ rediffmail.com

\begin{abstract}
Child Sexual Abuse (CSA) is a global problem and leads to many long-term serious complications to the abused child. Children do not disclose the abuse readily for many reasons which lead to a delay in reporting to healthcare setup. In many cases due to this delayed presentation, the examiner cannot find any physical signs of abuse; however, Child Forensic Interviewing (CFI) may give vital clues regarding the abuse and the perpetrator(s). The western countries have already established scientific protocols and techniques to conduct CFI, but in India till now it is an untouched topic. This review article addresses the importance of making interview models and techniques, and also discusses available guidelines, for effective execution of CFI in accordance with the POCSO Act, 2012.
\end{abstract}

Keywords: Child forensic interviewing, Child sexual abuse, POCSO Act, Child advocacy centres, Interview models.

\section{Introduction}

Child Sexual Abuse (CSA) is a widespread problem, and throughout the world, the incidence is in the rising phase. ${ }^{1}$ For a long time, except rape, other non-penetrative sexual abuses of children were not recognised as a criminal offence in India. Because of this reason, most of the CSA cases were gone unnoticed, and it was thought to be rare and only related to the people of poor socioeconomic status. In the past few years, the efforts of social activists and NonGovernmental Organisations (NGOs) to address various types of child abuses made the Indian government to realise the gravity and spectrum of this problem and the importance of taking strict measures. In 2012 the Indian government enacted new legislation called the Protection of Children from Sexual Offences (POCSO) Act. $^{2}$ Despite the introduction of POCSO Act and also increasing caseload, the bitter truth is that India is not yet ready for dealing with child abuse cases effectively.

CSA is a unique problem because the perpetrator is often a close relative or a known person, the abuse may be for weeks, months or years and due to fear, innocence, family values and social restrictions the child may not reveal it timely. So, in many cases, at the time of presenting to a hospital, the child will neither show any physical signs of abuse nor will reveal any history of the same. In this situation, Child Forensic Interviewing (CFI) provides vital clues regarding the crime and perpetrator(s). In western countries particularly in the United States, many models of CFI have already been developed, and the selection of a model depends on the interviewer, agencies and jurisdiction. CFI is a new concept for India and at present no special training programs and specialist exist for the same. This review article addresses the importance of making interview models and techniques, and also reviews the available recommendations in India and United States for the effective execution of CFI

\section{Child Sexual Abuse (CSA)}

World Health Organisation (WHO) defines Child Sexual Abuse as "the involvement of a child in sexual activity that he or she does not fully comprehend, is unable to give informed consent to, or for which the child is not developmentally prepared and cannot give consent, or that violates the laws or social taboos of society. Child sexual abuse is evidenced by this activity between a child and an adult or another child who by age or development is in a relationship of responsibility, trust or power, the activity is intended to gratify or satisfy the needs of the other person. This may include but is not limited to:

1. The inducement or coercion of a child to engage in any unlawful sexual activity;

2. The exploitative use of a child in prostitution or other unlawful sexual practices;

3. The exploitative use of children in pornographic performance and materials". 3

In most of the CSA cases, the perpetrator is often a close relative or a known person, and for that reason, many children do not disclose readily fearing the consequences. ${ }^{4}$ The disclosure is a long-term process starting in the form of a physical problem or a behavioural change. The child may face severe health consequences like Chronic abdominal pain, irritable bowel syndrome, chronic pelvic pain, menstrual irregularities, anxiety, depression, low selfesteem, symptoms of post-traumatic stress disorder, increase sexual behaviour, substance abuse, loss of social competence, etc. ${ }^{5,6}$

\section{Child Forensic Interview Definition}

Office of Juvenile Justice and Delinquency Prevention, U.S Department of Justice defines Child Forensic Interview as "a developmentally sensitive and legally sound method of gathering factual information regarding allegations of abuse or exposure to violence. This interview is conducted by a competently trained, neutral professional utilising research 
and practice-informed techniques as part of a larger investigative process". 7 The forensic history is different from medical history and the purpose is to assist the law enforcement to find the perpetrators and to take appropriate judicial actions.

\section{History}

In the 1980s, United States started to realise increasing child sexual abuse and neglect cases which concerned general public, health care workers and law enforcement agencies. At that time one of the most critical issues in dealing child abuse cases was obtaining relevant information from the child and caregivers. Many clinical histories submitted to the court by healthcare workers did not serve the purpose. The Courts started to raise doubts about the usability and credibility of the interviews conducted to extract the information from the child victims. US Government felt that interviewing children for medico-legal purpose needs specially trained professionals. Following that after so many researches on CFI techniques, child mental development, behavioural changes and other related topics, professionals have gained a good knowledge about interview techniques to extract maximum relevant information from children about the sexual abuse. The protocols and techniques have undergone many revisions and improvement through the continued research, peer reviews and practical experiences while dealing the cases. It is now a standard practice in the U.S that the CFI is conducted only by the professionals having formal forensic interview training. ${ }^{7}$

\section{CFI Settings}

In the United States, child abuse problems are handled by Child Advocacy Centers (CAC) which provide two primary services - Forensic Interviews and Sexual Assault Forensic Exams. The evidence and information gathered are shared with law enforcement officials, Child abuse investigators, juvenile officers and other appropriate authorities. Other services provided are training and technical assistance for medical personnel for child abuse examinations, peer review of medical exam findings and providing expert witness in CSA cases. The National Children's Alliance (NCA) mandate these CACs to provide a child-friendly setting that makes the child feel comfortable, private, physically and mentally safe. ${ }^{8}$ The setup should have at least 2 to 3 separate rooms; a reception where relatives, family members can wait, a room for police or other investigating officers and a separate room with attached washroom for forensic interviews and examination of child victims. The room should be painted in warm colours, must have child-sized furniture, toys and artworks of nonfantasy nature. ${ }^{9}$ The more comfortable and feel private the child is, the more information is likely to be revealed. So it is suggested that the healthcare institutes in India should have a separate setup with all these facilities to deal with CSA cases effectively. All the services should be given under one roof to prevent the harassment to the already traumatised child.

\section{CFI Models}

Many models of forensic interviews have been developed in the United States, and the selection of a model depends on the interviewer, agencies and jurisdiction. Discussing different interview models is beyond the scope of this article, and some examples are given below.

1. National Institute of Child Health and Human Development (NICHD) - Forensic Interview Protocol. ${ }^{9}$

2. ChildFirst Forensic Interviewing Protocol. ${ }^{10}$

3. The National Children's Advocacy Center's Forensic Interview Structure. ${ }^{11}$

4. The American Professional Society on the Abuse of Children's Practice Guidelines. ${ }^{12}$

Although these models are different from each other, they all have the common steps like rapport-building phase, substantive phase and closure phase. In rapport-building phase, the interviewer introduces himself and explains the child about interview instructions, documentation methods and makes him or her to realise the importance of revealing the truth.

Substantive phase focuses on getting a narrative description of abuse from the child, clarifying the facts and testing of alternative hypothesis. In closure phase, interviewer discusses the topic that is not related to the abuse. He addresses educational, social, emotional needs of the child and about safety plans in future. ${ }^{9-12}$

\section{Need for CFI Protocols and Experts in India}

CSA is a rampant problem in India, and this country has the world's largest number of CSA cases. One in every ten children is undergoing sexual abuse at any point of time, every $155^{\text {th }}$ minute an underage girl (less than sixteen years) raped and for every thirteenth hour, a child age less than ten is raped. ${ }^{13}$ To deal with increasing child sexual abuse problems, the Government of India brought a special law namely 'The Protection of Children from Sexual Offences (POCSO) Act, 2012" and it came into force with effect from $14^{\text {th }}$ November 2012. This act is a comprehensive law that defines various sexual offences like sexual assault, sexual harassment, pornography, child trafficking and its punishment. It also safeguards the interest of the child and giving protection at every stage of judicial proceedings like reporting, recording evidence, medical examination, investigation and trail. ${ }^{14}$ Subsequently, in September 2013, the Ministry of Women and Child Development has released guidelines for the use of professionals and experts under the POCSO Act, 2012 which includes CFI protocol. ${ }^{15}$ However, at ground level till now, no adequately trained professionals and no standardised interview models suitable for Indian set up are available for CFI.

In CSA cases, interviewing a child victim is the most important; at the same time more difficult task for investigating officers. Law enforcement agencies largely depend on the psychiatrists for this purpose because of their ability to deal with children and making rapport with them. But the mental health workers in India are primarily trained for therapeutic purpose and most of the time their medical history and interviews are inappropriate and cannot be 
considered as a forensic evidence. The court also finds difficulties in interpreting this medical history for the purpose of criminal investigations as it lacks many forensic details. For the effective implementation of POCSO Act, there should be a multidisciplinary approach involving forensic medicine experts, psychiatrists, paediatricians and gynaecologists; particularly for the purpose of CFI, forensic medicine experts and psychiatrists should be trained well, as in the majority of cases many relevant medico-legal facts can be derived from the interview itself.

\section{Recommendations for conducting CFI in a hospital set up in accordance with model guidelines given by Ministry of Women and Child Development under POCSO Act, 2012 and CFI techniques followed in western countries}

A child victim of sexual abuse can be presented to a healthcare institute for forensic examination in the following ways:

1. A child can be presented by a child care authority or the police with a request for medical examination.

2. Referral from a health care professional under the suspicion of child sexual abuse after noticing physical injuries or behavioural changes.

3. Family members or neighbours present the child with the allegation of sexual abuse.

Interview and forensic examination of the abused child should be conducted in accordance with section $164 \mathrm{~A}$ of the code of Criminal Procedure, 1973 and Section 27 of the POCSO Act 2012. The POCSO Act, 2012 defines Child as "any person less than eighteen years of age. Interviewing the children needs a careful approach. It is better to conduct CFI as soon as the child present to the healthcare setup. As time passes the chances of getting credible information from the child diminishes. The child may lose interest in reporting or he/she can be manipulated or threatened. If the child is not stable and needs emergency care, the forensic examination can be delayed till he or she becomes stable; the primary goal is to give medical care to the abused child rather than a forensic investigation. No hospital or healthcare setup can demand any legal or magisterial requisition or any documents to provide medical care, conducting a forensic interview and examination. Respect the privacy of the child; the interview room should be private and free from any distractions like horn sound, television, phones, crowd, drawings or toys of a fantasy nature. Health care institutes should create POCSO centres for children similar to One Stop Crisis Centres (OSCC) which deals with rape cases. POCSO centres should provide all services like forensic interview, medical examination, treatment, police \& NGO related services, safe custody of the child etc. under one roof.

The interview should be conducted by a woman registered medical practitioner preferably a psychiatrist or a forensic medicine expert trained for this purpose. Interview should be conducted in the presence of child's parents or any other trusted persons. In case the parents or any other trusted persons are not available or if the interviewer doubts that the accompanying person might have indulged in abuse, the head of institute or healthcare setup should nominate a women staff to accompany the child during the interview process.

Before proceeding with forensic interview and examination of a child written informed consent should be taken from the parents, relatives, caregivers or a legal custodian. The examiner should explain all the steps of forensic examination like forensic interview, physical examination, collecting medical and forensic samples, to the child and family members in a language they understand easily. If the Child is more than twelve years, the interviewer may take written consent from the child in addition to getting it from parents or legal guardians. If parents or other legal custodians refuse to give consent, but the doctor suspects sexual abuse strongly, then the child protection authority or the head of the healthcare setup should be called to take over the custody of the child for being forensically interviewed and examined. Because in some cases even parents or close relatives may be the culprits and their refusal should not be considered as a reason for not examining the child. ${ }^{3,10,11}$ When a medical practitioner is suspecting child abuse, it is his or her duty to inform the appropriate authority (e.g., nearest police post) even without any allegation from the parents or relatives. POCSO Act 2012 mandates the reporting of child sexual abuse. If a doctor fails to do that, he or she may get sixmonth imprisonment. Doctors don't need to wait for the consent from parents or legal guardians for reporting CSA cases.

Document the exact time and date of commencement of Forensic Interview and who were present along with the child during the interview process. Document all the questions asked and child's answer in his/her own words. The best alternative method is to digitally record the complete interview process. This digital material can be used in the court of law as an exhibit and also for doctor's review and research purposes. Avoid wearing white coat and introduce yourself as a helping person. A child-friendly environment should be created before proceeding with the interview. Don't jump straight to the questions about abuse. Ask general questions first that invite the child to talk and try to establish rapport and confidence. Do not show any surprise, disbelief or do not suggest your feelings like "I know how difficult it is to accept", "it is so terrible to hear this news" etc., and also do not make false promises. Use mainly open-ended questions like "Tell me why you are here today?" Tell me everything you know" Allow the child narrate his or her story regarding the abuse. Give a break if the child is not following you. During the narration, ask follow-up, simple questions like "then what happened?" "tell me more". Leading questions should be avoided initially. After the child's narration to the open-ended prompts, leading prompts can be used to extract forensically relevant missing details like the date, time, place of incidence, about perpetrator(s), sexual acts like penetration etc. Always put age-appropriate questions and avoid uncomfortable questions. The child may feel uncomfortable 
to talk about private parts with the adult interviewer, so using child's language to indicate body parts will make the child more comfortable. The interviewer can also use drawings to discuss body parts. Clarify the identity of the perpetrator, whether it happened with other children, whether it happened for one time or multiple times, whether the child disclosed the abuse to anyone. Be careful about the child not being exposed again to perpetrator during your interview and examination, only allow the person in whom the child has trust. When a child is interviewed by a team of doctors, only one doctor should ask questions to the child. Rest of them should simply observe and document the child's answer. Do not discuss the case in front of the child. Address the needs of the child such as hunger, thirst or urge to go toilet during the interview. Take enough time for the complete evaluation of the child. Avoid taking multiple interviews by multiple persons, because repeated exposure to the same questions and multiple adult members will scare the child, and he or she may undergo mental assault for the second time. If the child has some physical or mental disability, take help from an interpreter, translator or special educator.

Medical practitioners should periodically review their protocols, the type of questions asked in the interview and its effectiveness. Over the years by doing repeated research and from practical experiences while dealing with cases, a scientific, child-friendly, result-oriented interview protocols suitable for Indian setup should be created to deal CSA cases effectively.

\section{Conclusion}

Interviewing a child to get forensic evidence is a crucial part of dealing CSA cases, and it needs special skill. In India, the current practice of forensic interview in CSA cases is not satisfactory. Ministry of Women and Child Development, Government of India, has issued model guidelines under section 39 of POCSO Act, 2012 regarding forensic interviewing in child abuse cases. With these guidelines and scientific research conducted in other countries, healthcare professionals in India should start to practice proper CFI models. Interview models suitable for Indian society should be formulated through the periodic peer reviews and research activities. These measures will help our nation to deal with Child sexual abuse problems effectively.

\section{Source of Funding: None.}

\section{Declaration of Interests: None}

\section{Conflicts of Interest: None.}

\section{References}

1. Singh MM, Parsekar SS, Nair SN. An epidemiological overview of child sexual abuse. J Family Med Primary Care 2014;3(4):430.
2. Belur J, Singh BB. Child sexual abuse and the law in India: a commentary. Crime Sci 2015;4(1):26.

3. World Health Organization, 2003. Guidelines for medico-legal care of victims of sexual violence.

4. Kacker L, Mohsin N, Dixit A, Varadan S, Kumar P. Study on child abuse: India, 2007. Ministry of Women and Child Development, Government of India; 2007.

5. Banyard VL, Williams LM, Siegel JA. The long-term mental health consequences of child sexual abuse: An exploratory study of the impact of multiple traumas in a sample of women. J Traumatic Stress 2001;14(4):697-715.

6. South Eastern Centre Against Sexual Assault \& Family Violence. "Indicators of Child Sexual Abuse." South Eastern Centre Against Sexual Assault \& Family Violence. South Eastern Centre Against Sexual Assault \& Family Violence, n.d. Web. $15 ; 2017$.

7. Newlin, Chris, Linda C. Steele, Andra Chamberlin, Jennifer Anderson, Julie Kenniston, Amy Russell, Heather Stewart and Viola Vaughan-Eden. 2015. CFIing: Best Practices. Juvenile Justice Bulletin, U.S. Department of Justice. [cited 2018 Mar 2]. http://www.ojjdp.gov/pubs/248749.pdf.

8. National Children's Alliance; Standards for Accredited Members, 2017 Edition. [cited 2018 Mar 4]

Availableonline:https://vtt.ovc.ojp.gov/ojpasset/Documents/OS _NCA_Standards_Child_Advocacy_Centers-508.pdf.

9. NICHD; Revised Investigative Interview Protocol Version 2014. [cited 2018 Mar 4]. Available online:

http://nichdprotocol.com/wp-

content/uploads/2013/03/RevisedProtocolTMWH2final.pdf.

10. Gundersen National Child Protection Training Center; The Child First Forensic Interview Protocol 2018. [cited 2018 Mar 10]. Available online: https://www.gundersenhealth.org/app/files/public/2529/CFFIP. pdf.

11. National Children's Advocacy Center Huntsville; Alabama CFI Structure, 2012.[cited 2018 Mar 10]. Available online: http://www.nationalcac.org/wpcontent/uploads/2016/10/NCAC-Child-Forensic-InterviewStructure.pdf.

12. APSAC Taskforce. The American Professional Society on the Abuse of Children (APSAC); Forensic Interviewing in Cases of Suspected Child Abuse,2012. [cited 2018 Mar 10]. Available online: https://www.apsac.org/guidelines.

13. Singh MM, Parsekar SS, Nair SN. An epidemiological overview of child sexual abuse. J Family Med Primary Care 2014;3(4):430.

14. Ministry of Law and Justice (Legislative Department), Government of India, New Delhi; The Protection Of Children From Sexual Offences Act, 2012. [cited 2018 Apr 5]. Available online: http://wcd.nic.in/sites/default/files/childprotection31072012.pd f.

15. Ministry of Women and Child Development, Government of India; Model Guidelines under Section 39 of the Protection of Children from Sexual Offences Act, 2012, 2013. [cited Apr 5]. Available online: http://wcd.nic.in/sites/default/files/POCSOModelGuidelines.pdf.

How to cite this article: Senthil KR, Bansal YS, Priyadarshini NC, Child forensic interviewing - An important investigative tool in child sexual abuse cases:, Indian $J$ Forensic Community Med 2019;6(2):45-8. 